\title{
283.
}

\section{ON A THEOREM RELATING TO HOMOGRAPHIC FIGURES.}

[From the Quarterly Journal of Pure and Applied Mathematics, vol.' III. (1860), pp. 177-180.]

The following theorem is not new, but I do not remember where to find it:

Given any two homographic figures; there exists in the first figure a point $S$, which is the focus of an (ellipse or hyperbola, say an) ellipse $\Sigma$; and in the second figure a point $S^{\prime}$, which is the focus of a (hyperbola or ellipse, say a) hyperbola $\Sigma^{\prime}$, such that the points $S, S^{\prime \prime}$ and the conics $\Sigma, \Sigma^{\prime}$ correspond to each other, and the conics $\Sigma, \Sigma^{\prime}$ are so related that the foci and vertices of the one may be superimposed upon the vertices and foci of the other. Moreover the perpendiculars from $S, S^{\prime}$ upon corresponding tangents of $\Sigma, \Sigma^{\prime}$ will be equal.

Write

$$
x: y: 1=\alpha \xi+\beta \eta+\gamma: \alpha^{\prime} \xi+\beta^{\prime} \eta+\gamma^{\prime}: \alpha^{\prime \prime} \xi+\beta^{\prime \prime} \eta+\gamma^{\prime \prime},
$$

then $l, m, \lambda, \mu$ may be so determined that $(x-l)+i(y-m)$ shall vanish with $(\xi-\lambda)+i(\eta-\mu)$, for if we write

$$
\begin{array}{ll}
J=\alpha-l \alpha^{\prime \prime}, & J^{\prime}=\alpha^{\prime}-m \alpha^{\prime \prime}, \\
K=\beta-l \beta^{\prime \prime}, & K^{\prime}=\beta^{\prime}-m \beta^{\prime \prime}, \\
L=\gamma-l \gamma^{\prime \prime}, & L^{\prime}=\gamma^{\prime}-m \gamma^{\prime \prime},
\end{array}
$$

we have

$$
(x-l)+i(y-m)=\frac{\left(J+i J^{\prime}\right)\{(\xi-\lambda)+i(\eta-\mu)\}}{\alpha^{\prime \prime} \xi+\beta^{\prime \prime} \eta+\gamma^{\prime \prime}} ;
$$

and therefore

$$
\begin{aligned}
& K+i K^{\prime}=i\left(J+i J^{\prime}\right), \\
& L+i L^{\prime}=\left(J+i J^{\prime}\right)(\lambda+i \mu),
\end{aligned}
$$


that is

or

$$
K=-J, \quad K^{\prime}=J
$$

$$
\begin{array}{r}
l \beta^{\prime \prime}+m \alpha^{\prime \prime}=\alpha^{\prime}+\beta, \\
-l \alpha^{\prime \prime}+m \beta^{\prime \prime}=-\alpha+\beta^{\prime},
\end{array}
$$

whence also

$$
\begin{aligned}
l\left(\alpha^{\prime \prime 2}+\beta^{\prime 2}\right) & =\alpha^{\prime} \beta^{\prime \prime}-\alpha^{\prime \prime} \beta^{\prime}+\alpha \alpha^{\prime \prime}+\beta \beta^{\prime \prime}, \\
m\left(\alpha^{\prime \prime 2}+\beta^{\prime \prime 2}\right) & =\alpha^{\prime \prime} \beta-\alpha \beta^{\prime \prime}+\alpha \alpha^{\prime}+\beta \beta^{\prime},
\end{aligned}
$$

which determine $l, m$; and then $\lambda, \mu$ are given by

$$
\lambda+i \mu=\frac{L+i L^{\prime}}{J+i J^{\prime}}
$$

but more simply as follows, viz. remarking that if $c=\beta^{\prime} \gamma^{\prime \prime}-\beta^{\prime \prime} \gamma^{\prime}$, \&c., so that

$$
\xi: \eta: 1=a x+a^{\prime} y+a^{\prime \prime}: b x+b^{\prime} y+b^{\prime \prime}: c x+c^{\prime} y+c^{\prime \prime}
$$

then we have

$$
\begin{aligned}
& \lambda\left(c^{2}+c^{\prime 2}\right)=b c^{\prime}-b^{\prime} c+a c+a^{\prime} c^{\prime}, \\
& \mu\left(c^{2}+c^{\prime 2}\right)=c a^{\prime}-c^{\prime} a+b c+b^{\prime} c^{\prime}
\end{aligned}
$$

the equations $x=l, y=m$ give the point $S$ and $\xi=\lambda, \eta=\mu$ the point $S^{\prime}$. The values of $J, J^{\prime}$ are

$$
J=\frac{-c \alpha^{\prime \prime}-c^{\prime} \beta^{\prime \prime}}{\alpha^{\prime \prime 2}+\beta^{\prime \prime 2}}, \quad J^{\prime}=\frac{-c^{\prime} \alpha^{\prime \prime}+c \beta^{\prime \prime}}{\alpha^{\prime \prime 2}+\beta^{\prime \prime 2}}
$$

and therefore

if

$$
J^{2}+J^{\prime 2}=\frac{c^{2}+c^{\prime 2}}{\alpha^{\prime \prime 2}+\beta^{\prime \prime 2}}=\frac{k^{2}}{K^{2}}
$$

$$
k=\sqrt{ }\left(c^{2}+c^{\prime 2}\right), \quad K=\sqrt{ }\left(\alpha^{\prime \prime 2}+\beta^{\prime \prime 2}\right):
$$

we have therefore

$$
\sqrt{ }\left\{(x-l)^{2}+(y-m)^{2}\right\}=\frac{k}{K} \frac{\sqrt{ }\left\{(\xi-\lambda)^{2}+(\eta-\mu)^{2}\right\}}{\alpha^{\prime \prime} \xi+\beta^{\prime \prime} \eta+\gamma^{\prime \prime}} .
$$

Consider now the expression

$$
(x-l) \cos 9+(y-m) \sin 9-\varpi,
$$

which made equal to 0 would be the equation of a line the perpendicular distance of which from $S$ is $\varpi$, and which distance is inclined at an angle 9 to the axis of $x$; then

$$
\begin{aligned}
& (x-l) \cos 9+(y-m) \sin 9=\frac{1}{2}[(\cos 9-i \sin 9)\{(x-l)+i(y-m)\} \\
& +(\cos 9+i \sin 9)\{(x-l)-i(y-m)\}] \text {, } \\
& =\frac{1}{2\left(\alpha^{\prime \prime} \xi+\beta^{\prime \prime} \eta+\gamma^{\prime \prime}\right)}\left[(\cos \vartheta-i \sin 9)\{(\xi-\lambda)+i(\eta-\mu)\}\left(J+i J^{\prime}\right)\right. \\
& \left.+(\cos 9+i \sin 9)\{(\xi-\lambda)-i(\eta-\mu)\}\left(J-i J^{\prime}\right)\right] \\
& \text { 56-2 }
\end{aligned}
$$




$$
\begin{aligned}
& =\frac{k}{2\left(\alpha^{\prime \prime} \xi+\beta^{\prime \prime} \eta+\gamma^{\prime \prime}\right) K}\left[\left\{\cos \left(\Im-9_{0}\right)-i \sin \left(9-\Im_{0}\right)\right\}\{(\xi-\lambda)+i(\eta-\mu)\}\right. \\
& \left.+\left\{\cos \left(9-9_{0}\right)+i \sin \left(\Im-9_{0}\right)\right\}\{(\xi-\lambda)-i(\eta-\mu)\}\right] \\
& =\frac{k}{\left(\alpha^{\prime \prime} \xi+\beta^{\prime \prime} \eta+\gamma^{\prime \prime}\right) K}\left\{(\xi-\lambda) \cos \left(\Im-\Im_{0}\right)+(\eta-\mu) \sin \left(\Im-\Im_{0}\right)\right\},
\end{aligned}
$$

where $J, J^{\prime}$ have been replaced by

$$
J=\frac{k}{K} \cos \Im_{0}, \quad J^{\prime}=\frac{k}{K} \sin \vartheta_{0} .
$$

And putting besides

we have more simply

$$
9-9=\theta
$$

$$
(x-l) \cos 9+(y-m) \sin \vartheta=\frac{k}{\left(\alpha^{\prime \prime} \xi+\beta^{\prime \prime} \eta+\gamma^{\prime \prime}\right) K}\{(\xi-\lambda) \cos \theta+(\eta-\mu) \sin \theta\},
$$

whence

$(x-l) \cos 9+(y-m) \sin 9-\pi$

$$
\begin{aligned}
& =\frac{k}{\left(\alpha^{\prime \prime} \xi+\beta^{\prime \prime} \eta+\gamma^{\prime \prime}\right) K}\left\{(\xi-\lambda) \cos \theta+(\eta-\mu) \sin \theta-\frac{\varpi K}{k}\left(\alpha^{\prime \prime} \xi+\beta^{\prime \prime} \eta+\gamma^{\prime \prime}\right)\right\} \\
& =\frac{k}{\left(\alpha^{\prime \prime} \xi+\beta^{\prime \prime} \eta+\gamma^{\prime \prime}\right) K}\left\{(\xi-\lambda)\left(\cos \theta-\frac{\varpi K \alpha^{\prime \prime}}{k}\right)+(\eta-\mu)\left(\sin \theta-\frac{\varpi K \beta^{\prime \prime}}{k}\right)-\frac{\varpi K}{k}\left(\alpha^{\prime \prime} \lambda+\beta^{\prime \prime} \mu+\gamma^{\prime \prime}\right)\right\} .
\end{aligned}
$$

Write now

$$
\alpha^{\prime \prime}=K \cos j, \beta^{\prime \prime}=K \sin j ; \quad \mathrm{a}=\frac{k}{K^{2}}, \quad \mathrm{~b}=\frac{1}{K}\left(\alpha^{\prime \prime} \lambda+\beta^{\prime \prime} \mu+\gamma^{\prime \prime}\right)
$$

we have

$$
(x-l) \cos 9+(y-m) \sin 9 \quad-\sigma
$$

$$
\begin{aligned}
& =\frac{K b}{\alpha^{\prime \prime} \xi+\beta^{\prime \prime} \eta+\gamma^{\prime \prime}}\left\{\frac{\mathrm{a}}{\mathrm{b}}\left(\cos \theta-\frac{\varpi \cos j}{\mathrm{a}}\right)(\xi-\lambda)+\frac{\mathrm{a}}{\mathrm{b}}\left(\sin \theta-\frac{\varpi \sin j}{\mathrm{a}}\right)(\eta-\mu)-\varpi\right\} \\
& =\frac{K \mathrm{~b}}{\alpha^{\prime \prime} \xi+\beta^{\prime \prime} \eta+\gamma^{\prime \prime}}\{(\xi-\lambda) \cos \phi+(\eta-\mu) \sin \phi-\varpi\},
\end{aligned}
$$

that is

$$
\begin{aligned}
& (x-l) \cos 9+(y-m) \sin 9-\varpi \quad(*) \\
& \quad=\frac{K b}{\alpha^{\prime \prime} \xi+\beta^{\prime \prime} \eta+\gamma^{\prime \prime}}\{(\xi-\lambda) \cos \phi+(\eta-\mu) \sin \phi-\varpi\},
\end{aligned}
$$

where it will be noticed that

and

$$
(x-l) \cos 9+(y-m) \sin 9-\sigma=0,
$$

$$
(\xi-\lambda) \cos \phi+(\eta-\mu) \sin \phi-\sigma=0,
$$


are lines in the first figure and in the second figure, corresponding to each other and at the same distance $\approx$ from the points $S, S^{\prime \prime}$ respectively. Call these lines $T$ and $T^{\prime \prime}$.

But the relations between $\theta, \phi$, $\approx$ are given by

$$
\begin{aligned}
& \varpi \cos j=\mathrm{a} \cos \theta-\mathrm{b} \cos \phi, \\
& \varpi \sin j=\mathrm{a} \sin \theta-\mathrm{b} \sin \phi,
\end{aligned}
$$

or, by changing the fixed axes from which $\theta, \phi$ are respectively measured,

$$
\begin{aligned}
& \varpi=a \cos \theta-b \cos \phi, \\
& 0=a \sin \theta-b \sin \phi .
\end{aligned}
$$

Now in these equations $\varpi$ is the perpendicular distance from $S$ upon the line $(x-l) \cos 9+(y-m) \sin 9-\sigma=0$ and $\theta$ (which only differs from 9 by a constant angle) is the inclination of this perpendicular to a certain fixed line; $\varpi$ is also the perpendicular distance of the line $(\xi-\lambda) \cos \phi+(\eta-\mu) \sin \phi-\sigma=0$ from the point $S^{\prime}$, and $\phi$ is the inclination of this perpendivular to a fixed line. Eliminating successively $\phi$ and $\theta$, we have

$$
\begin{aligned}
& \varpi=a \cos \theta-\sqrt{ }\left(b^{2}-a^{2} \sin ^{2} \theta\right), \\
& \varpi=-b \cos \phi+\sqrt{ }\left(a^{2}-b^{2} \sin ^{2} \phi\right),
\end{aligned}
$$

or, as these equations may also be written,

$$
\begin{aligned}
& \varpi^{2}-2 \varpi a \cos \theta+a^{2}-b^{2}=0, \\
& \varpi^{2}+2 \varpi b \cos \phi+b^{2}-a^{2}=0 .
\end{aligned}
$$

Suppose $\mathrm{a}>\mathrm{b}$, the former equation shows that the line $T$ is a tangent to a certain hyperbola, and the latter equation shows that the line $T^{\prime}$ is a tangent to a certain ellipse, and it is easily seen that, taking for the transverse axes the lines from which the angles $\theta$ and $\phi$ are respectively measured, the equation of the hyperbola is

$$
\frac{x^{2}}{\mathrm{~b}^{2}}-\frac{y^{2}}{\mathrm{a}^{2}-\mathrm{b}^{2}}=1
$$

and that of the ellipse is

$$
\frac{\xi^{2}}{a^{2}}+\frac{y^{2}}{a^{2}-b^{2}}=1
$$

which are the conics $\Sigma, \Sigma^{\prime}$ referred to in the enunciation. And if the second conic is superimposed upon the first in such manner that the coordinates $\xi, \eta$ may belong to the same axes with $x, y$; then the two conics will have the assumed relation, viz. the foci of either conic will coincide with the vertices of the other conic. 